\title{
Thermal transport and phonon focusing in complex molecular crystals: $A b$ initio study of polythiophene
}

\author{
Peishi Cheng ${ }^{\circledR}$, Nina Shulumba, and Austin J. Minnich* \\ Division of Engineering and Applied Science, California Institute of Technology, Pasadena, California 91125, USA
}

(Received 17 May 2019; revised manuscript received 31 July 2019; published 17 September 2019)

\begin{abstract}
Thermally conductive molecular crystals are of fundamental interest because they are unlike typical complex crystals, which conduct heat poorly owing to their large phonon scattering phase space. While molecular crystals with high thermal conductivity in the range of tens of $\mathrm{Wm}^{-1} \mathrm{~K}^{-1}$ have been known experimentally for decades, their intrinsic upper limits for thermal conductivity are unclear. Ab initio methods that have been successfully applied to simple crystals have proved difficult to adapt to molecular crystals due to quantum nuclear motion and their complex primitive cells. Here, we report the thermal transport properties of crystalline polythiophene with 28 atoms per primitive cell using an $a b$ initio approach that rigorously includes finite-temperature anharmonicity and quantum nuclear effects. The calculated room temperature thermal conductivity is $198 \mathrm{Wm}^{-1} \mathrm{~K}^{-1}$ along the chain axis, a high value that arises from exceptional phonon focusing along the chain for both acoustic and optical branches for nearly all wave vectors and despite short lifetimes in the picosecond range. Our finding, along with other recent $a b$ initio studies of polyethylene, suggests that the intrinsic upper bounds for the chain axis thermal conductivity of polymer crystals may exceed $100 \mathrm{Wm}^{-1} \mathrm{~K}^{-1}$.
\end{abstract}

DOI: 10.1103/PhysRevB.100.094306

\section{INTRODUCTION}

Crystalline polymers with high thermal conductivity exceeding $10 \mathrm{Wm}^{-1} \mathrm{~K}^{-1}$ are intriguing materials because of their potential use in thermal management applications [1] and because complex crystals with many atoms per unit cell tend to be thermally insulating [2]. The microscopic origin of insulating behavior in complex materials is well understood in terms of the phonon scattering phase space, defined as the allowed space of energy- and momentum-conserving threephonon interactions [3,4]. A crystal which has many atoms per primitive cell and thus many vibrational degrees of freedom usually has a larger scattering phase space than that of a simpler crystal, leading to shorter lifetimes and lower thermal conductivity.

However, early experiments have shown that semicrystalline polymers can achieve thermal conductivities comparable to those of some metals in the direction of oriented polymer chains [5,6], indicating that molecular crystals can be intrinsically good thermal conductors despite their complex atomic structure. The thermal conductivity of oriented semicrystalline polyethylene has been studied for decades [7-12] with the highest values reported for nanofibers [13,14]. Thermal conductivities greater than $60 \mathrm{Wm}^{-1} \mathrm{~K}^{-1}$ in the draw direction were recently reported for macroscopic ribbon samples of polyethylene [15,16]. Even for more complex molecular crystals with up to 48 atoms per unit cell, thermal conductivities on the order of tens of $\mathrm{Wm}^{-1} \mathrm{~K}^{-1}$ have been reported [17].

The high uniaxial thermal conductivity in crystalline polymers can be partially attributed to phonon focusing in which

*Corresponding author: aminnich@ caltech.edu the phonon group velocity is not aligned with the wave vector, leading to enhanced energy flow along certain crystallographic directions $[18,19]$. This focusing phenomena can be understood as a necessary consequence of anisotropic bonding. Stronger covalent bonds in one crystal axis compared to the others result in higher group velocities in that axis, leading to ellipsoidal isofrequency surfaces in the Brillouin zone. Since the group velocity is the gradient of frequency with respect to wave vector, an ellipsoidal surface will have group velocity vectors preferentially oriented along the short axis of the ellipse. However, many previous works studying phonon focusing as it pertains to thermal conductivity make simplifying assumptions, such as the use of a Debye model for only low-wave-vector, low-frequency acoustic modes [20,21]. Because of these assumptions, it is difficult to determine the intrinsic upper bounds of thermal conductivity in polymer crystals or assess the role phonon focusing plays in setting this upper bound.

Instead, atomistic simulations have been used to study the upper limits of thermal conductivity. Molecular dynamics simulations have yielded insights into the thermal conductivity trends with respect to morphology [22-24] and molecular characteristics [25], but the use of semiempirical potentials yields qualitative discrepancies in the predicted thermal conductivity values $[26,27]$. The $a b$ initio lattice dynamics approach to thermal conductivity [28] can overcome these challenges, and this approach has been used for polyethylene [29] and other quasi-one-dimensional (quasi-1D) complex materials $[30,31]$. Recently, the temperature-dependent effective potential (TDEP) method has been adapted for polymer crystals [32], enabling the inclusion of zero-point motion [32,33] and finite-temperature anharmonicity [34-37]. TDEP includes these effects by sampling the Born-Oppenheimer energy surface at the temperature of interest using thermal amplitudes 
that include the additional displacement from quantum nuclear motion. The correct quantum statistics for nuclear motion are important for molecular crystals given the large number of hydrogen atoms with zero-point energies corresponding to $1000 \mathrm{~K}$. A recent study of using the TDEP method on a polymer crystal reported that the intrinsic thermal conductivity of polyethylene is $164 \mathrm{Wm}^{-1} \mathrm{~K}^{-1}$ and that using the incorrect classical statistics would lead to a 50\% deviation [32].

Crystalline polythiophene is an example of a complex polymer crystal. It has attracted interested in its substituted form, poly(3-hexylthiophene) (P3HT), for both its thermal $[38,39]$ and electrical properties [40]. Experimentally, a relatively high thermal conductivity of $4 \mathrm{Wm}^{-1} \mathrm{~K}^{-1}$ was reported for amorphous aligned chains of unsubstituted polythiophene [41]. However, a first-principles investigation into the interplay of phonon focusing and scattering that determines the rigorous upper bound of thermal conductivity in crystalline polythiophene remains lacking.

Here, we report an ab initio study of the thermal transport properties of crystalline unsubstituted polythiophene (PT) using TDEP that rigorously includes finite-temperature anharmonicity and quantum nuclear effects. We calculate the room-temperature thermal conductivity along the chain to be $198 \mathrm{Wm}^{-1} \mathrm{~K}^{-1}$ at $300 \mathrm{~K}$, higher than that of many metals and semiconductors. We find that phonon focusing of both acoustic and optical branches, at nearly all wave vectors and beyond where continuum approximations are valid, is a key factor in the high thermal conductivity of PT, despite short lifetimes for the optical branches in the picosecond range. Our findings suggest that polymer crystals may have exceptionally high intrinsic upper limits of thermal conductivity along the chain axis, rivaling those of covalent solids, due to the generality of the phonon focusing mechanism.

\section{THEORY}

We compute the lattice dynamical and thermal transport of properties using the TDEP method, which we briefly review. The $a b$ initio approach to thermal conductivity is based on computing the interatomic force constants using density functional theory (DFT). From the interatomic force constants, all the relevant phonon properties can be calculated using lattice dynamics and the Boltzmann transport equation. In the TDEP method, atoms in a supercell of the crystal are given thermal amplitudes that correspond to a canonical ensemble of the desired temperature, accounting for finitetemperature anharmonicity and allowing for the inclusion of zero-point motion $[32,33]$. The displacements of the atoms and the resulting forces, as calculated from DFT, compose a set of linear equations which are linked by the interatomic force constants. Solving these linear equations with symmetry constraints yields the irreducible set of force constants which represent the best fit of the Born-Oppenheimer energy surface sampled at the temperature of interest. With these inputs, the Boltzmann transport equation can be solved using an iterative method to obtain the thermal conductivity [28]. We use a 5.0 - $\AA$ cutoff for the second-order interatomic force constants which yields convergence of the vibrational free energy within $0.1 \mathrm{meV}$ per atom. We use a 3.0- $\AA$ cutoff for the third-order interatomic force constants. For a grid of comparable density, using an increased third-order cutoff of $3.3 \AA$ yields only a reduction of $4 \%$ in the thermal conductivity.

We use the VASP implementation of DFT [42-45] to calculate the forces. The plane-wave energy cutoff was $800 \mathrm{eV}$ with a reciprocal space mesh of $3 \times 3 \times 3$ to sample the Brillouin zone. We incorporate van der Waals interactions with a nonlocal density functional [46-48] (commonly denoted vdw-DF) that captures long-range correlation and has been tested for the polyethylene crystal [49]. Others have studied the performance of various nonlocal functionals for polymer crystals [50] and found that this functional, and an updated version, performed best in estimating the $a$ and $b$ lattice parameters. We note that the choice of functional can affect the computed value of the thermal conductivity [51]. Our choice of functional was motivated by the desire to include van der Waals interaction in a parameter-free way. Eight iterations were required to obtain self-consistent force constants. A single iteration is composed of the extraction of the force constants from the thermalized supercell and generation of the thermal displacements for the supercells used in the successive iteration. The supercell was composed of a $2 \times 2 \times 2$ repetition of the orthorhombic unit cell for a total of 224 atoms.

The Boltzmann transport equation was solved iteratively on a $18 \times 18 \times 18$ grid in the Brillouin zone with a tetrahedron integration method for energy conservation. The included scattering mechanisms are intrinsic anharmonic phonon-phonon scattering and isotopic scattering [52] from the natural isotope distribution. Convergence of the thermal conductivity was verified by calculating thermal conductivity for various $q$-grid densities and plotting the inverse of the grid density with thermal conductivity to extrapolate to infinite grid density. If we use an anisotropic $q$ grid where the chain axis has a higher grid density, extrapolation yields a value of $201 \mathrm{Wm}^{-1} \mathrm{~K}^{-1}$. If we use an isotropic grid, extrapolation yields a value of $195 \mathrm{Wm}^{-1} \mathrm{~K}^{-1}$. We report the value using the $18 \times 18 \times 18$ grid since it is obtained using the densest grid calculated, and the value lies within the two extrapolated values.

\section{RESULTS}

We applied TDEP to obtain the vibrational and thermal tranport properties of unsubstituted PT. The structure of the PT unit cell is given in Figs. 1(a)-1(c). Figure 1(a) shows the chains extending along the $z$ axis. The atoms in each chain are covalently bonded, while van der Waals bonding occurs between chains. Figure 1(b) shows the orthorhombic primitive cell which contains two such chains. The chains from adjacent cells are shown for reference. Figure 1(c) shows a single PT chain composed of the thiophene repeat units; a primitive cell contains two thiophene repeat units per chain. Our calculated lattice parameters from the relaxed structure are $a=$ $7.84 \AA, b=5.86 \AA$, and $c=7.85 \AA$ for an orthorhombic unit cell. The lattice parameters from experiment are $a=7.80 \AA$, $b=5.55 \AA$, and $c=8.03 \AA$ also for an orthorhombic unit cell [53], corresponding to a mean absolute relative error of $2.78 \%$.

The computed phonon dispersion for PT is given in Fig. 1(d). The dispersion contains 84 branches corresponding 
(a)

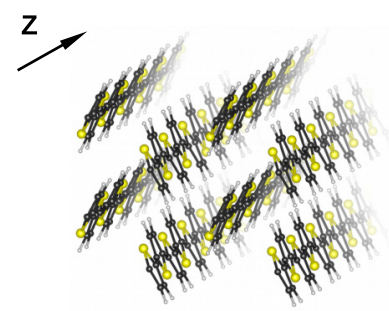

(b)

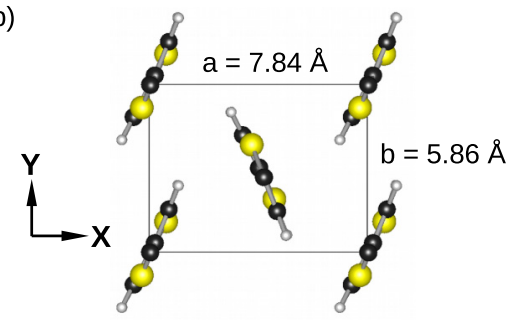

(c)

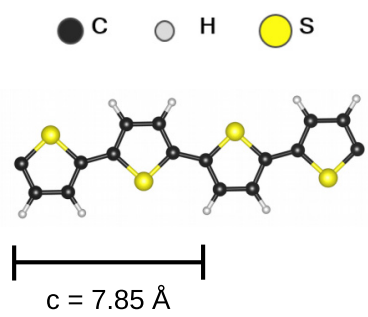

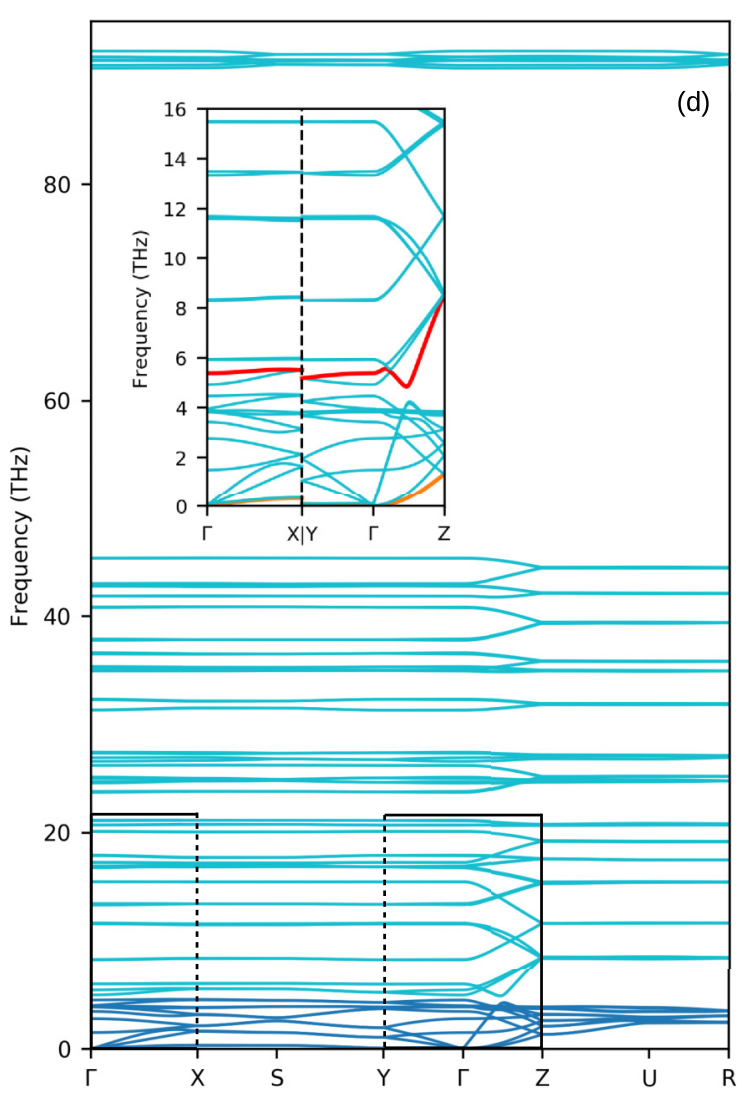

FIG. 1. The structure and dispersion of polythiophene (PT). (a) Structure of a PT crystal with chains extending in the $z$ axis. (b) View of the $x y$ plane of the unit cell. There are two chains in each unit cell. Chains from the adjacent cell are shown. (c) Single-polymer chain of PT showing that the length of unit cell in the chain axis is two thiophene chemical repeat units. (d) Calculated phonon dispersion of PT, showing numerous branches. The dark blue and light blue branches correspond to the acoustic-like and optic modes, respectively, as seen in Fig. 2. Inset: The phonon dispersion along symmetry directions from $\Gamma$. The branches for which isoenergy contours are plotted later are highlighted in orange [Fig. 4(a)] and red [Fig. 4(b)].

to the degrees of freedom of the 28 -atom primitive cell. The branches are highly dispersive in the $\Gamma-Z$ direction up to nearly $50 \mathrm{THz}$, whereas the branches are far less dispersive in the $\Gamma-X$ and $\Gamma-Y$ directions, becoming nearly dispersionless above $5 \mathrm{THz}$.

Such a complex crystal would be expected to have a low thermal conductivity if considering only the phononscattering phase space, which would be large given the numerous branches. For instance, the primitive cell of tetrahedrite contains 29 atoms per cell and has a thermal conductivity of around $1 \mathrm{Wm}^{-1} \mathrm{~K}^{-1}$ [54]. We calculate the chain-axis thermal conductivity of PT to be $198 \mathrm{Wm}^{-1} \mathrm{~K}^{-1}$ at room temperature. This value is higher than both the room-temperature thermal conductivity of silicon and the $a b$ initio thermal conductivity of the simpler polyethylene crystal, which has only 12 atoms per unit cell [32]. The cross-chain thermal conductivity along the $x$ and $y$ axes are calculated as 8.3 and $7.3 \mathrm{Wm}^{-1} \mathrm{~K}^{-1}$ at room temperature, respectively, a substantial thermal anisotropy which is expected for these elastically anisotropic polymer crystals.

The thermal conductivity versus temperature, as shown in Fig. 2(a), was calculated by solving the Boltzmann transport equation at each temperature using the force constants generated at $300 \mathrm{~K}$. While other works have independently calculated force constants for a grid of temperature and volume, such a calculation is computationally expensive for our system due to the size and complexity of the PT crystal. We calculated force constants on a grid of volumes at a single temperature $(300 \mathrm{~K})$ and determine the equilibrium volume at this temperature by minimizing the Gibbs free energy. We then used the force constants at the equilibrium volume to calculate thermal conductivity at other temperatures. Given that the force constants are fixed, the trend shows the expected decrease of thermal conductivity with increasing temperature as our calculation includes only isotope and intrinsic anharmonic scattering.

Figure 2(b) shows the spectral thermal conductivity versus phonon frequency. Isolating purely acoustic modes below $5 \mathrm{THz}$ is difficult because there is significant overlap in frequencies with some optical modes, as was also observed for polyethylene [32]. Thus, we denote the modes below $5 \mathrm{THz}$ as acoustic-like modes, and all modes above $5 \mathrm{THz}$ as optical modes. Greater than $96 \%$ of the thermal conductivity is due to phonon modes with frequencies below $16 \mathrm{THz}$, even though modes exist up to $92 \mathrm{THz}$. Around two thirds of the heat is conducted from the acoustic-like modes, with the remainder carried by the optical modes.

We next examine how the lifetimes and group velocities compare between PT and $\mathrm{Si}$. The comparison with $\mathrm{Si}$, an isotropic crystal with a lower but same order of magnitude 

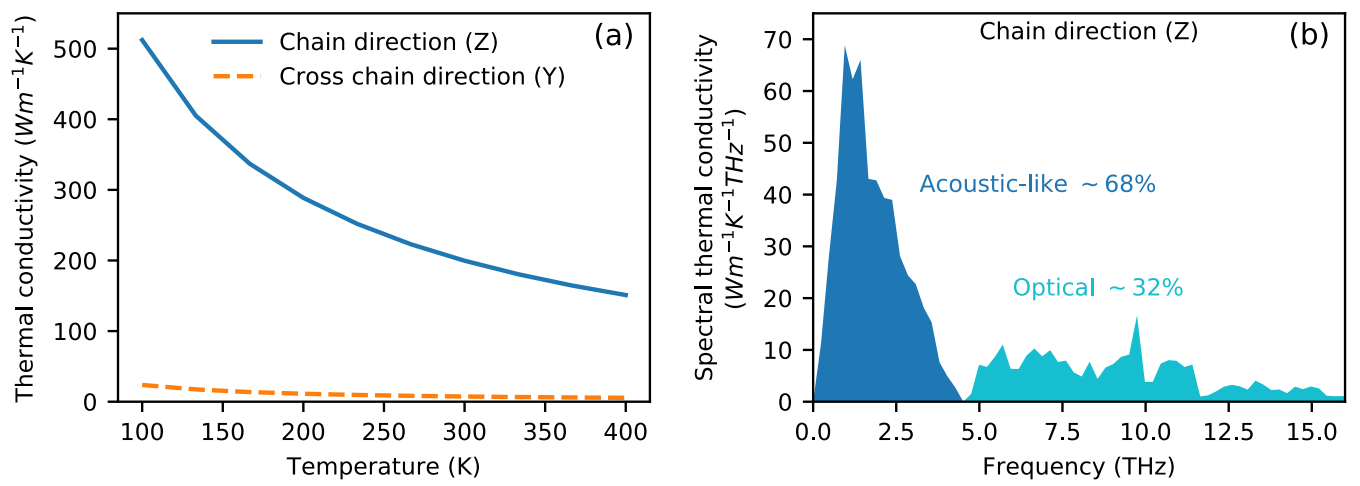

FIG. 2. Thermal conductivity vs temperature and spectral thermal conductivity. (a) Thermal conductivity along the chain (blue solid line) and along the $y$ axis perpendicular to the chains (orange dashed line) vs temperature using force constants obtained at $300 \mathrm{~K}$. The polymer chains are oriented along the $z$ axis (blue line). The chain-axis thermal conductivity is $198 \mathrm{Wm}^{-1} \mathrm{~K}^{-1}$ at room temperature. (b) Spectral thermal conductivity vs phonon frequency along the chain axis. The contributions from acoustic-like modes and optic modes are highlighted in dark blue and light blue, respectively.

thermal conductivity, will reveal the importance of phonon focusing. We calculated the thermal conductivity of Si using the phonon properties generated by TDEP in Ref. [55]. The phonon lifetimes versus frequency for PT and Si are shown in Fig. 3(a). The results show that the lifetimes in PT are an order of magnitude lower than in Si over nearly the entire frequency range of $0-16 \mathrm{THz}$ despite PT having the higher thermal conductivity. We can quantitatively assess how much heat is carried by individual modes over the entire spectrum of phonon lifetimes by examining thermal conduc- tivity accumulation plots with respect to lifetime, shown in Fig. 3(b). We observe that long lifetime modes in Si contribute significantly to the thermal conductivity, with over half of the thermal conductivity coming from modes with lifetimes greater than $50 \mathrm{ps.} \mathrm{In} \mathrm{contrast,} \mathrm{in} \mathrm{PT} \mathrm{there} \mathrm{are} \mathrm{almost} \mathrm{no}$ modes contributing to thermal conductivity that have lifetimes greater than $50 \mathrm{ps}$. This comparison suggests that the lifetimes for these complex crystals are of the order expected of a crystal with a complex unit cell and thus high scattering rates.
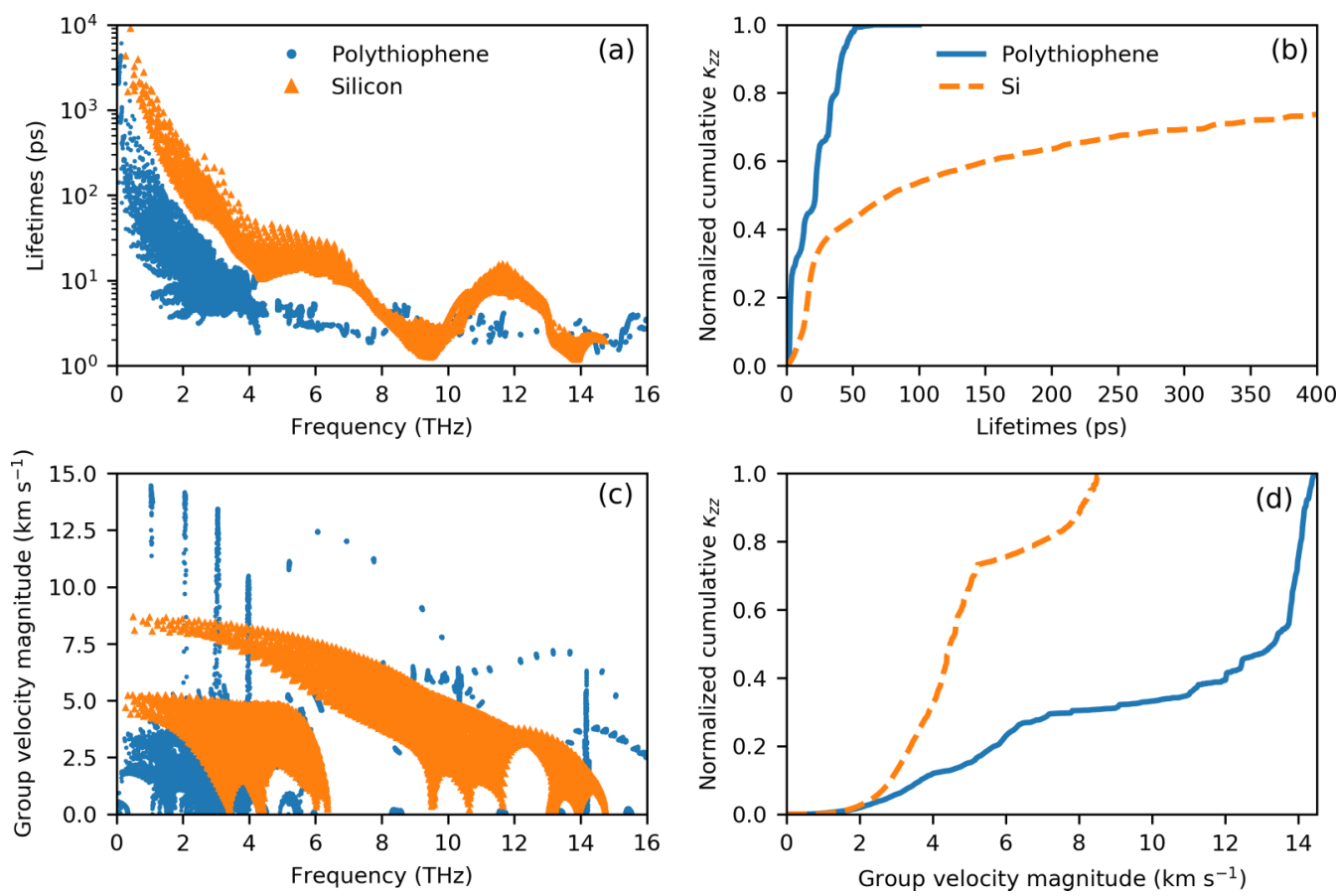

FIG. 3. Phonon lifetimes and group velocities of PT and Si. (a) Phonon lifetimes vs frequency for PT (blue dots) and Si (orange triangles). (b) Thermal conductivity accumulation function vs lifetime. The thermal conductivity of PT is due almost entirely to modes with lifetimes less than $50 \mathrm{ps}$, in contrast to $\mathrm{Si}$ which has more than half of its thermal conductivity from modes with lifetimes longer than 100 ps. (c) Phonon group velocities along the chain axis ( $z$ ) vs frequency of PT (blue dots) and Si (orange triangles). (d) Thermal conductivity accumulation function vs group velocity along the chain axis. Nearly two thirds of the heat in PT is carried by modes with group velocity larger than the maximum group velocity in $\mathrm{Si}$. 

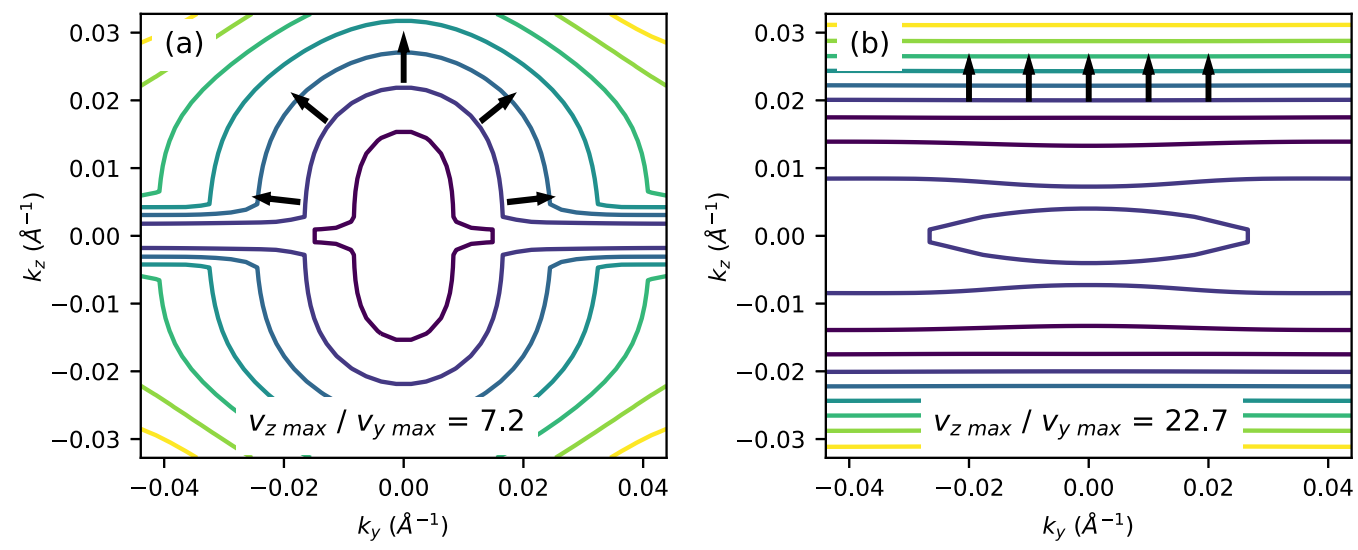

FIG. 4. Isoenergy contours in PT. (a) The isoenergy contours of an acoustic branch with an anisotropy in the maximum group velocity ratio of 7.2 in PT for the $k_{x}=0$ plane. The lines are contours of equal frequency. The arrows show the direction of the group velocity vector. The contours are similar to those Ref. [20] corresponding to the quasitransverse acoustic branch. (b) The isoenergy contours of an optical branch with a much larger anisotropy ratio of 22.7. The group velocity of nearly all the modes are pointed along the chain axis. We observe a similar effect in other planes in the Brillouin zone for this branch and for other optical branches with a large anisotropy ratio.

Next, we present the group velocity magnitude versus frequency in Fig. 3(c). We observe that PT possesses modes with higher group velocity than those in $\mathrm{Si}$. To determine whether a higher group velocity magnitude by itself can explain the high thermal conductivity of PT, we compute the rms group velocity $\left(\sqrt{\sum_{\lambda} v_{\lambda}^{2} / N_{\lambda}}\right.$ where $\lambda$ denotes phonon mode given by a unique combination of wavevector and polarization, $v$ denotes group velocity, and $N_{\lambda}$ denotes the total number of phonon modes). For PT, the value is $4.33 \mathrm{~km} \mathrm{~s}^{-1}$ below 18 $\mathrm{THz}$, which is the frequency range containing $99 \%$ of the thermal conductivity. The value for $S i$ is $3.46 \mathrm{~km} \mathrm{~s}^{-1}$ below 13 $\mathrm{THz}$, which is the corresponding frequency range containing 99\% of the heat-carrying modes. From these results alone, and without taking phonon focusing into account, how PT can have a higher thermal conductivity than Si despite having an order of magnitude lower lifetimes and only a slight increase in the rms group velocity is not obvious.

PT is highly anisotropic and thus phonon focusing is expected to occur. A way to assess the strength of phonon focusing is to compare the rms group velocity in the chain direction to the rms group velocity magnitude. In PT, this rms group velocity in the chain direction $(0-18 \mathrm{THz})$ is $4.29 \mathrm{~km} \mathrm{~s}^{-1}$, which is indeed very close to the rms group velocity magnitude of $4.33 \mathrm{~km} \mathrm{~s}^{-1}$. For $\mathrm{Si}$, the RMS group velocity in the chain direction $(0-13 \mathrm{THz})$ is $2.00 \mathrm{~km} \mathrm{~s}^{-1}$, which is far from the rms group velocity magnitude of $3.46 \mathrm{~km} \mathrm{~s}^{-1}$.

The effect of phonon focusing is also evident in Fig. 3(d). Over half of the heat in PT is carried by modes with group velocity greater than $12 \mathrm{~km} \mathrm{~s}^{-1}$ because the highest group velocity modes correspond to the acoustic-like modes below $5 \mathrm{THz}$, which possess group velocity vectors oriented along the chain as well as the longest lifetimes in PT. However, optical modes also contribute substantially due to phonon focusing. While these modes possess the shortest lifetimes, as seen in the frequency range above $5 \mathrm{THz}$ in Fig. 3(a), phonon focusing allows these modes to overcome their low lifetimes to still contribute $32 \%$ of the total thermal conductivity. As a comparison, for $\mathrm{Si}$ we find that once the lifetimes become comparable to those in PT in the frequency range of
8-10 THz there is negligible contribution to thermal conductivity, despite the group velocities in that range being near the maximum for $\mathrm{Si}$, because of the lack of phonon focusing.

To further analyze the influence of phonon focusing, we plot isoenergy contours. We note that in contrast to previous work, our isoenergy contours are generated by calculating the dynamical matrix for a dense grid in the Brillouin zone using the $a b$ initio force constants and subsequently plotting lines of equal frequency for a given plane in the Brillouin zone. Thus, they are valid beyond the typical frequency and wave vector ranges of isoenergy contours derived from continuum elasticity. The group velocity is the gradient of the frequency with respect to wave vector, so the group velocity vector is always normal to an isoenergy contour.

Figure 4(a) plots the isoenergy contours of a branch with a ratio of the largest group velocity along the chain axis to the largest group velocity along the cross-chain axis of 7.2, corresponding to the orange branch in the inset of Fig. 1(d). The contours are similar to those in Ref. [20] corresponding to the quasitransverse acoustic branch. On the other hand, Fig. 4(b) shows the isoenergy contours of an optical branch with an anisotropy ratio of 22.7 , corresponding to the red branch in the inset of Fig. 1(d). The contours reveal an exceptional degree of phonon focusing such that even for vibrations with wave vectors pointing almost completely orthogonal to the direction of the chain, the vibrational energy is still transported in the direction of the chain. We observe this effect throughout the Brillouin zone, irrespective of the plane for which the isoenergy contours are plotted. This phenomenon resembles the degree of focusing calculated for quasilongitudinal acoustic branches $[20,21]$, but in PT the optical branches are also focused.

We quantitatively assess the importance of phonon focusing for the chain axis thermal conductivity by calculating the thermal conductivity of an isotropic version of PT in which the group velocity and wave vector are parallel. Note that only the direction of the group velocity is changed, but not the magnitudes of the group velocities and lifetimes. We find that the thermal conductivity of this isotropic crystal would be 


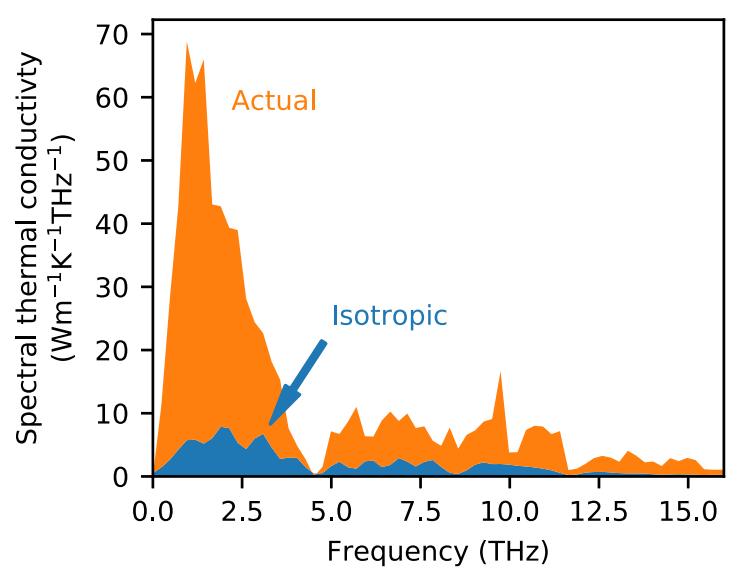

FIG. 5. The spectral thermal conductivity vs frequency in the case where the group velocities are parallel to their wave vector (blue) compared to the actual case (orange). Phonon focusing underlies the high thermal conductivity of PT, particularly for modes below $5 \mathrm{THz}$.

only $34 \mathrm{Wm}^{-1} \mathrm{~K}^{-1}$, confirming that the anisotropy is the key factor which underlies the high thermal conductivity. In fact, if we calculate the other extreme, where the group velocity vectors of every mode is pointing along the chain direction, the thermal conductivity increases by only $7.5 \%$. We performed this computation for the polyethylene crystal as well, finding an "isotropic" thermal conductivity for polyethylene of $67 \mathrm{Wm}^{-1} \mathrm{~K}^{-1}$, less than half of the actual calculated value. The extreme case of perfect focusing in the chain direction would yield only a $3.2 \%$ increase.

To further emphasize this conclusion, we plot the spectral thermal conductivity for the hypothetical isotropic PT as seen in Fig. 5. In the frequency range where the acoustic-like modes previously contributed over $100 \mathrm{Wm}^{-1} \mathrm{~K}^{-1}$ (68\% of the original thermal conductivity), there is now very little contribution, even though this frequency range contains modes with the largest group velocities and longest lifetimes in PT. In contrast, if we calculate this isotropic thermal conductivity for silicon, it is unchanged from the actual value.

\section{DISCUSSION}

We now discuss our results in the context of prior work on polymer crystals. First, many works use an average lifetimes or average mean free paths to analyze thermal transport in polymer crystals due to computational or experimental limitations $[17,20]$. Using our ab initio calculations, we are able to assess the accuracy of these approximations. First, as in Fig. 3(b), we find that the distribution of lifetimes for phonons that conduct heat is narrow, between 0 and $50 \mathrm{ps,} \mathrm{so} \mathrm{that}$ assuming an average lifetime is in fact a reasonable estimate. However, the estimate of an average mean free path is less accurate because the shortest lifetime modes can contribute significantly to thermal transport due to phonon focusing, while modes with group velocity approaching the maximum value possess the longest lifetimes. Thus, the range of mean free paths relevant for heat transport spans $1 \mathrm{~nm}$ to $1 \mu \mathrm{m}$.

Second, we examine previous treatments of phonon focusing in anisotropic solids. Many previous works employed the Debye approximation because of the assumption of that acoustic phonons dominate the thermal transport [20,21]. Our work demonstrates that phonon focusing has a dramatic effect on both acoustic and optical phonons for nearly all phonons regardless of wave vector because dispersive optical modes persist throughout the thermal phonon frequencies. Consequently, future studies on thermal transport in polymers will have to be more cautious when interpreting results using simple models neglecting optical modes, since focusing allows optical phonons to contribute substantially to heat transport.

We note that the phonon focusing effect is expected to be more effective in quasi-1D materials compared to quasi-2D materials like graphite, where the group velocities in the $a b$ plane are similar because of covalent bonding between atoms in the plane, and thus the focusing largely exists only from anisotropy with the cross-plane direction. In the quasi-1D case, both cross-chain directions have weak van der Waals bonding while the chain direction has strong covalent bonds, resulting in the focusing of an additional dimension of phonon modes and an enhanced chain-axis thermal conductivity. Indeed, we find that for the polarization shown in Fig. 4(b), 87\% of the modes have a group velocity vector that points less than 10 deg away from the chain axis.

Finally, we consider our prediction for PT in comparison to the predicted and experimentally realized thermal conductivities of other crystalline polymers. Prior computational works reported a range of 8 to $100 \mathrm{Wm}^{-1} \mathrm{~K}^{-1}$ in polymer crystals lacking significant disorder [25] while the highest reported experimental values in macroscopic samples have ranged from 20 to around $65 \mathrm{Wm}^{-1} \mathrm{~K}^{-1}$ [15-17]. Our work, along with other recent ab initio studies [32], indicates that the upper bound for the chain axis thermal conductivity of polymer crystals is at least $160 \mathrm{Wm}^{-1} \mathrm{~K}^{-1}$. The steady progress in synthesis and associated increase in measured thermal conductivity in various material systems suggest that the predicted values from $a b$ initio calculations can serve as useful guiding references, highlighting the utility of parameter-free thermal conductivity calculations for the materials synthesis community. While defects and the difficulties of crystallizing polymers are substantial barriers to achieving high thermal conductivity, the generality of the phonon-focusing mechanism in suggests that there are some crystalline polymers that, if synthesized at sufficiently high quality, could have thermal conductivities exceeding $100 \mathrm{Wm}^{-1} \mathrm{~K}^{-1}$.

\section{SUMMARY}

We calculated the thermal conductivity of crystalline unsubstituted polythiophene from first-principles including quantum nuclear effects and finite-temperature anharmonicity. We obtain a thermal conductivity of $198 \mathrm{Wm}^{-1} \mathrm{~K}^{-1}$ for crystalline PT at room temperature. We find that exceptional phonon focusing of both acoustic and optical branches that affects nearly all of the modes in the Brillouin zone permits even modes with short, picosecond-range lifetimes to contribute to heat conduction. Our work indicates that the intrinsic upper bound for the chain axis thermal conductivity of polymer crystals may rival the values of metals and semiconductors, as phonon focusing occurs in any anisotropic crystal. 


\section{ACKNOWLEDGMENTS}

This work was supported by the Office of Naval Research under Award No. N00014-18-1-2101. This work used the Extreme Science and Engineering Discovery Environment
(XSEDE), which is supported by National Science Foundation Grant No. ACI-1548562. This work used Comet under XSEDE at the San Diego Supercomputing Center through allocation TG-PHY160029 and the Navy DoD Supercomputing Resource Center (DSRC).
[1] X. Xu, J. Chen, J. Zhou, and B. Li, Adv. Mater. 30, 1705544 (2018).

[2] G. J. Snyder and E. S. Toberer, Nat. Mater. 7, 105 (2008).

[3] A. Ward, D. A. Broido, D. A. Stewart, and G. Deinzer, Phys. Rev. B 80, 125203 (2009).

[4] L. Lindsay, D. A. Broido, and T. L. Reinecke, Phys. Rev. Lett. 111, 025901 (2013).

[5] C. L. Choy, Polymer 18, 984 (1977).

[6] L. Piraux, M. Kinany-Alaoui, J. P. Issi, D. Begin, and D. Billaud, Solid State Commun. 70, 427 (1989).

[7] S. Burgess and D. Greig, J. Phys. C: Solid State Phys. 8, 1637 (1975).

[8] H. Kilian and M. Pietralla, Polymer 19, 664 (1978).

[9] C. Choy, W. Luk, and F. Chen, Polymer 19, 155 (1978).

[10] C. L. Choy, F. C. Chen, and W. H. Luk, J. Polym. Sci.: Polym. Phys. Ed. 18, 1187 (1980).

[11] D. B. Mergenthaler, M. Pietralla, S. Roy, and H. G. Kilian, Macromolecules 25, 3500 (1992).

[12] C. L. Choy, Y. W. Wong, G. W. Yang, and T. Kanamoto, J. Polym. Sci. Part B 37, 3359 (1999).

[13] S. Shen, A. Henry, J. Tong, R. Zheng, and G. Chen, Nat. Nano. 5, 251 (2010).

[14] R. Shrestha, P. Li, B. Chatterjee, T. Zheng, X. Wu, Z. Liu, T. Luo, S. Choi, K. Hippalgaonkar, M. P. d. Boer, and S. Shen, Nat. Commun. 9, 1664 (2018).

[15] S. Ronca, T. Igarashi, G. Forte, and S. Rastogi, Polymer 123, 203 (2017).

[16] Y. Xu, D. Kraemer, B. Song, Z. Jiang, J. Zhou, J. Loomis, J. Wang, M. Li, H. Ghasemi, X. Huang, X. Li, and G. Chen, Nat. Commun. 10, 1771 (2019).

[17] X. Wang, V. Ho, R. A. Segalman, and D. G. Cahill, Macromolecules 46, 4937 (2013).

[18] M. Pietralla, J. Comput.-Aided Mater. Des. 3, 273 (1996).

[19] B. Taylor, H. J. Maris, and C. Elbaum, Phys. Rev. B 3, 1462 (1971).

[20] M. Pietralla, R. M. Weeger, and D. B. Mergenthaler, Z. Phys. B Condens. Matter 77, 219 (1989).

[21] Z. Chen, Z. Wei, Y. Chen, and C. Dames, Phys. Rev. B 87, 125426 (2013).

[22] J. Liu and R. Yang, Phys. Rev. B 81, 174122 (2010).

[23] T. Luo, K. Esfarjani, J. Shiomi, A. Henry, and G. Chen, J. Appl. Phys. 109, 074321 (2011).

[24] H. Ma and Z. Tian, Appl. Phys. Lett. 110, 091903 (2017).

[25] T. Zhang, X. Wu, and T. Luo, J. Phys. Chem. C 118, 21148 (2014).

[26] B. Ni, T. Watanabe, and S. R. Phillpot, J. Phys. Condens. Matter 21, 084219 (2009).

[27] A. Henry and G. Chen, Phys. Rev. Lett. 101, 235502 (2008).

[28] D. A. Broido, M. Malorny, G. Birner, N. Mingo, and D. A. Stewart, Appl. Phys. Lett. 91, 231922 (2007).

[29] X. Wang, M. Kaviany, and B. Huang, Nanoscale 9, 18022 (2017).
[30] Q. Zhang, C. Liu, X. Liu, J. Liu, Z. Cui, Y. Zhang, L. Yang, Y. Zhao, T. T. Xu, Y. Chen, J. Wei, Z. Mao, and D. Li, ACS Nano 12, 2634 (2018).

[31] T. Pandey, C. A. Polanco, V. R. Cooper, D. S. Parker, and L. Lindsay, Phys. Rev. B 98, 241405(R) (2018).

[32] N. Shulumba, O. Hellman, and A. J. Minnich, Phys. Rev. Lett. 119, 185901 (2017).

[33] N. Shulumba, O. Hellman, and A. J. Minnich, Phys. Rev. B 95, 014302 (2017).

[34] O. Hellman, I. A. Abrikosov, and S. I. Simak, Phys. Rev. B 84, 180301(R) (2011).

[35] O. Hellman and I. A. Abrikosov, Phys. Rev. B 88, 144301 (2013).

[36] O. Hellman and D. A. Broido, Phys. Rev. B 90, 134309 (2014).

[37] A. H. Romero, E. K. U. Gross, M. J. Verstraete, and O. Hellman, Phys. Rev. B 91, 214310 (2015).

[38] Y. Xu, X. Wang, J. Zhou, B. Song, Z. Jiang, E. M. Y. Lee, S. Huberman, K. K. Gleason, and G. Chen, Sci. Adv. 4, eaar3031 (2018).

[39] M. Muñoz Rojo, J. Martín, S. Grauby, T. Borca-Tasciuc, S. Dilhaire, and M. Martin-Gonzalez, Nanoscale 6, 7858 (2014).

[40] B. Russ, A. Glaudell, J. J. Urban, M. L. Chabinyc, and R. A. Segalman, Nat. Rev. Mater. 1, 16050 (2016).

[41] V. Singh, T. L. Bougher, A. Weathers, Y. Cai, K. Bi, M. T. Pettes, S. A. McMenamin, W. Lv, D. P. Resler, T. R. Gattuso et al., Nat. Nano. 9, 384 (2014).

[42] G. Kresse and J. Furthmüller, Phys. Rev. B 54, 11169 (1996).

[43] G. Kresse and J. Furthmüller, Comput. Mater. Sci. 6, 15 (1996).

[44] G. Kresse and D. Joubert, Phys. Rev. B 59, 1758 (1999).

[45] G. Kresse and J. Hafner, Phys. Rev. B 48, 13115 (1993).

[46] M. Dion, H. Rydberg, E. Schröder, D. C. Langreth, and B. I. Lundqvist, Phys. Rev. Lett. 92, 246401 (2004).

[47] J. Klimeš, D. R. Bowler, and A. Michaelides, Phys. Rev. B 83, 195131 (2011).

[48] G. Román-Pérez and J. M. Soler, Phys. Rev. Lett. 103, 096102 (2009).

[49] J. Kleis, B. I. Lundqvist, D. C. Langreth, and E. Schröder, Phys. Rev. B 76, 100201(R) (2007).

[50] T. H. Pham, R. Ramprasad, and H.-V. Nguyen, J. Chem. Phys. 144, 214905 (2016).

[51] A. Jain and A. J. H. McGaughey, Comput. Mater. Sci. 110, 115 (2015).

[52] S.-I. Tamura, Phys. Rev. B 27, 858 (1983).

[53] Z. Mo, K. B. Lee, Y. B. Moon, M. Kobayashi, A. J. Heeger, and F. Wudl, Macromolecules 18, 1972 (1985).

[54] X. Lu, D. T. Morelli, Y. Xia, F. Zhou, V. Ozolins, H. Chi, X. Zhou, and C. Uher, Adv. Energy Mater. 3, 342 (2013).

[55] D. S. Kim, O. Hellman, J. Herriman, H. L. Smith, J. Y. Y. Lin, N. Shulumba, J. L. Niedziela, C. W. Li, D. L. Abernathy, and B. Fultz, Proc. Natl. Acad. Sci. USA 115, 1992 (2018). 